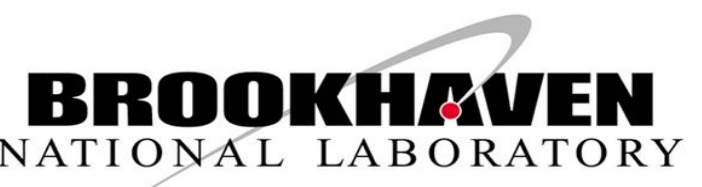

BNL- 112602-2016-JA

\title{
Intramolecular Singlet Fission in Oligoacene Heterodimers
}

Samuel N. Sanders, Elango Kumarasamy, Andrew B. Pun, Michael L. Steigerwald, Matthew Y. Sfeir and Luis M. Campos

Submitted to Angewandte Chemie

February 2016

Center for Functional Nanomaterials

Brookhaven National Laboratory

\author{
U.S. Department of Energy \\ USDOE Office of Science (SC), \\ Basic Energy Sciences (SC-22)
}

\footnotetext{
Notice: This manuscript has been authored by employees of Brookhaven Science Associates, LLC under Contract No. DE- SC0012704 with the U.S. Department of Energy. The publisher by accepting the manuscript for publication acknowledges that the United States Government retains a non-exclusive, paid-up, irrevocable, world-wide license to publish or reproduce the published form of this manuscript, or allow others to do so, for United States Government purposes.
} 


\section{DISCLAIMER}

This report was prepared as an account of work sponsored by an agency of the United States Government. Neither the United States Government nor any agency thereof, nor any of their employees, nor any of their contractors, subcontractors, or their employees, makes any warranty, express or implied, or assumes any legal liability or responsibility for the accuracy, completeness, or any third party's use or the results of such use of any information, apparatus, product, or process disclosed, or represents that its use would not infringe privately owned rights. Reference herein to any specific commercial product, process, or service by trade name, trademark, manufacturer, or otherwise, does not necessarily constitute or imply its endorsement, recommendation, or favoring by the United States Government or any agency thereof or its contractors or subcontractors. The views and opinions of authors expressed herein do not necessarily state or reflect those of the United States Government or any agency thereof. 


\title{
Intramolecular Singlet Fission in Oligoacene Heterodimers
}

\author{
Samuel N. Sanders, ${ }^{\S}$ Elango Kumarasamy, ${ }^{\S}$ Andrew B. Pun, Michael L. Steigerwald, Matthew Y. \\ Sfeir, * Luis M. Campos*
}

\begin{abstract}
In this Communication we investigate singlet fission $(S F)$ in heterodimers comprising a pentacene unit covalently bonded to another acene as we systematically vary the singlet and triplet pair energies. We find that these energies control the $S F$ process, where dimers undergo $S F$ provided that the resulting triplet pair energy is similar or lower in energy than the singlet state. In these systems the singlet energy is determined by the lower energy chromophore, and the rate of $S F$ is found to be relatively independent of the driving force. However, triplet pair recombination in these heterodimers follows the energy gap law. The ability to tune the energies of these materials provides a key strategy to study and design new SF materials - an important process for third generation photovoltaics.
\end{abstract}

Singlet exciton fission has attracted renewed interest in the last decade due to its potential to enhance power conversion efficiencies of single junction solar cells beyond the ShockleyQueisser Limit. ${ }^{[1]}$ The recent discovery of an efficient intramolecular singlet fission (iSF) process in conjugated polymers and small molecules has dramatically increased the quantity and variety of materials that exhibit this process. ${ }^{[2]}$ Moreover, the mechanism of triplet pair formation and decay may be quite different in dimers of oligoacenes relative to their monomer counterparts in the solid state, where singlet fission is an intermolecular process (xSF). For example, donor-acceptor polymers reported by our group are presumed to undergo SF via charge transfer (CT) states, similar to the leading hypothesis for the mechanism for solid state SF. ${ }^{[2 a, 3]}$ However, there is no intrinsic CT character in molecular dimers, yet they have been reported to undergo SF at faster rates than the donor-acceptor

[*] S. N. Sanders, Dr. E. Kumarasamy, A. B. Pun, Dr. M. L. Steigerwald, Prof. L. M. Campos

Department of Chemistry

Columbia University

3000 Broadway, MC3124

New York, NY 10027, USA

E-mail: Icampos@columbia.edu

Dr. M. Y. Sfeir

Center for Functional Nanomaterials

Brookhaven National Laboratory

Building 735

Upton, NY 11973, USA

${ }^{\S}$ These authors contributed equally to this work.

[**] This work was funded by the Office of Naval Research Young Investigator Program (Award N00014-15-1-2532), ACS Petroleum Research Fund, 3M Non-Tenured Faculty Award, and Cottrell Scholar Award. S.N.S. and A.B.P. thank the NSF GRFP (DGE 11-44155). This research used resources of the Center for Functional Nanomaterials, which is a U.S. DOE Office of Science Facility, at Brookhaven National Laboratory under Contract No. DESC0012704. We are grateful to the Nuckolls lab for use of their UV-vis spectrophotometer.

Supporting information for this article is available on the WWW under http://www.angewandte.org or from the author polymers. There are various important aspects that are still being actively investigated in terms of electronic structure, excited state energies and dynamics. ${ }^{[4]}$ Thus it is important to elucidate the mechanistic and energetic requirements for iSF in order to optimize the design of practical SF chromophores.
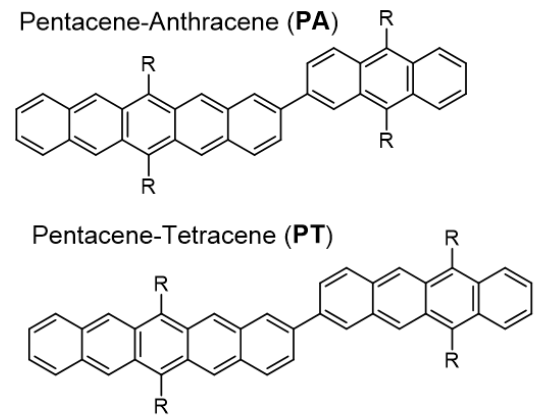

Monomer Excited State Energies Anthracene $\mathrm{S}_{1} \sim 2.7 \mathrm{eV}$ $\mathrm{T}_{1} \sim 1.8 \mathrm{eV}$ Tetracene $\mathrm{S}_{1} \sim 2.2 \mathrm{eV}$ $\mathrm{T}_{1} \sim 1.2 \mathrm{eV}$ Pentacene $\mathrm{S}_{1} \sim 1.9 \mathrm{eV}$ $\mathrm{T}_{1} \sim 0.8 \mathrm{eV}$ Hexacene $\mathrm{S}_{1} \sim 1.5 \mathrm{eV}$

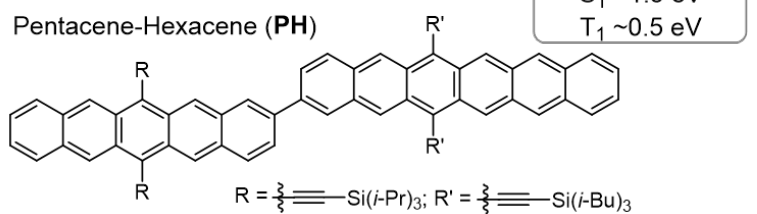

Scheme 1. Oligoacene heterodimers and the excited state energies of the respective monomers.

Molecular dimers made up of two covalently linked SF-capable monomers have long been considered as candidates for iSF. ${ }^{[5]}$ Early work on tetracene dimers showed low iSF yields, presumably because of the endothermicity of the iSF process or the connectivity employed. ${ }^{[6]}$ Pentacene dimers, on the other hand, have recently been reported to undergo iSF quantitatively. ${ }^{[2 \mathrm{~d}, 2 \mathrm{f}]}$ The potential to develop families of oligoacene dimers through systematic studies has motivated us to revisit the concept of singlet fission in oligoacene "mixtures", which was briefly explored in the 1970s when several groups studied crystals of one type of acene doped with another type of acene. ${ }^{[7]}$ In this vein, we explore iSF in asymmetric systems where two different oligoacene monomers are covalently linked (Scheme 1). This design feature allows us to systematically adjust the energetics of the iSF process, affecting both the driving force for singlet fission and the total energy of the resulting triplet pair. In these heterodimers, we demonstrate that the relevant singlet energy for iSF is given by the lower singlet state energy monomer, and the resulting triplet pair is a sum of the individual monomer triplets. Therefore, the fundamental equation for energy conservation is $\mathrm{E}\left(\mathrm{S}_{1}[\mathrm{X}]\right) \geq \mathrm{E}\left(\mathrm{T}_{1}[\mathrm{X}]\right)+\mathrm{E}\left(\mathrm{T}_{1}[\mathrm{Y}]\right)$, in a dimer comprising monomer $\mathrm{X}$ coupled to $\mathrm{Y}$. For example, the pentacene-tetracene heterodimer is nearly isoergic ( $\mathrm{S}_{1}[$ Pentacene $] \sim 1.9 \mathrm{eV}, \mathrm{T}_{1}$ [Pentacene $] \sim 0.8 \mathrm{eV}, \mathrm{T}_{1}$ [Tetracene] $\sim 1.2 \mathrm{eV}$ ), while our previously reported bipentacene molecule is exoergic $\left(\mathrm{S}_{1}[\right.$ Pentacene $]>2 \mathrm{xT}_{1}[$ Pentacene $\left.]\right) \cdot{ }^{[2 \mathrm{~d}, 8]}$ Furthermore, since pentacene-anthracene is significantly endoergic $\left(\mathrm{E}\left(\mathrm{S}_{1}[\right.\right.$ Pentacene $\left.]\right)<\mathrm{E}\left(\mathrm{T}_{1}[\right.$ Pentacene $\left.]\right)+\mathrm{E}\left(\mathrm{T}_{1}\right.$ [Anthracene $\left.\left.]\right)\right)$, it is not expected to undergo iSF. Here, we test this hypothesis and 
demonstrate that asymmetric dimers undergo fast and efficient iSF, provided that the singlet state is not significantly lower in energy than the resulting triplet pair. We also find that subsequent decay of the triplet pairs formed in iSF-capable heterodimers is primarily non-radiative, and it obeys the energy gap law for non-radiative recombination. ${ }^{[9]}$

In order to investigate singlet fission in oligoacene heterodimers, the molecules shown in Scheme 1 were synthesized via Suzuki coupling chemistry (See SI for details). ${ }^{[2 \mathrm{~d}]}$ The compounds are labeled as PA, PT, PH, where $\mathbf{P}, \mathbf{A}, \mathbf{T}$ and $\mathbf{H}$ refers to pentacene, anthracene, tetracene, and hexacene respectively. We also compare these results to bipentacene (BP), which we recently reported, in which two pentacenes are similarly covalently attached at the 2-position. The inclusion of tri-isopropylsilyl acetylene (TIPS), or in the case of hexacene, triisobutylsilyl acetylene groups (TIBS), renders these heterodimers soluble and relatively stable in solution. ${ }^{[10]}$

The steady state absorption spectra of the heterodimers show the characteristic features of both monomers (Figure 1A). In PA, $\mathbf{P T}$, and PH, we observe the prominent low-energy singlet transition peak associated with TIPS-pentacene $\left(\mathrm{S}_{1}[\mathbf{P}]\right)$ at $\sim 660$ $\mathrm{nm}$ and, respectively, its complement, with the anthracene peak $\left(\mathrm{S}_{1}[\mathrm{~A}]\right)$ at $\sim 470 \mathrm{~nm}$, the tetracene peak $\left(\mathrm{S}_{1}[\mathrm{~T}]\right)$ at $\sim 550 \mathrm{~nm}$, and the hexacene peak $\left(\mathrm{S}_{1}[\mathbf{H}]\right)$ at $\sim 750 \mathrm{~nm}$. A small redshift is observed in the dimers, relative to the monomer features. When coupling pentacenes at the 2-position, we also observe a highenergy feature in the ground state absorption. That feature, previously reported for 2,2' bipentacene (BP), is also observed in these compounds. ${ }^{[2 \mathrm{~d}]}$ It can be seen clearly in Figure 1A for PA, but this peak in PT and $\mathbf{P H}$ has been omitted for clarity (see SI for full spectra). This high-energy feature is specific to directly coupled acenes at the position shown, and does not correspond to a peak in the parent monomers.

We use broadband transient absorption spectroscopy (TAS) to understand the exciton dynamics in these molecules. Since we are probing the energetic requirements for iSF, the chromophores are pumped at the lower singlet energy selectively ( $\mathbf{P}$ transitions for PA and PT, and $\mathbf{H}$ transitions for $\mathbf{P H}$ ) to determine if iSF occurs without significant excess excitation energy. Figure 1B shows the resulting $2 \mathrm{D}$ plot of the spectral evolution of the transient absorption spectra as a function of time.

In the case of $\mathbf{P A}$, where iSF is expected to be significantly endothermic, we observe no significant spectral changes of the singlet state features. In fact, the photophysics of this heterodimer are similar to TIPS-pentacene, with a photoexcited singlet that decays with a $\sim 11.5$ ns time constant primarily through a radiative pathway (SI). The singlet lifetime is long enough to permit a small amount of triplet formation via intersystem crossing (ISC). By comparing the magnitude of the ground state bleach in the singlet and triplet manifold, we calculate a triplet yield of $\sim 10 \%$ giving an ISC time constant of 104 ns. $^{[8 c]}$ The triplet relaxation dynamics (SI) are similar to TIPS-pentacene as well, with a decay time of $17.4 \mu \mathrm{s}$. This result verifies that, in the case where energetics are not appropriate for iSF, no additional decay pathways are present in these compounds beyond the typical monomer excited state deactivation.

However, in PT and PH, singlet fission is roughly isoergic and exothermic, respectively. In these systems, TAS reveals dynamics similar to those observed in $\mathbf{B P}$, where the photoexcited singlet rapidly decays into a triplet signal in dilute solution, consistent with iSF. ${ }^{[2 \mathrm{~d}]}$ The triplet pair feature produced by iSF is dominated by the photoinduced absorption of the larger acene in each case, as the triplet absorption cross-section increases with increasing acene length. ${ }^{[8 \mathrm{a}, 8 \mathrm{c}, 11]}$
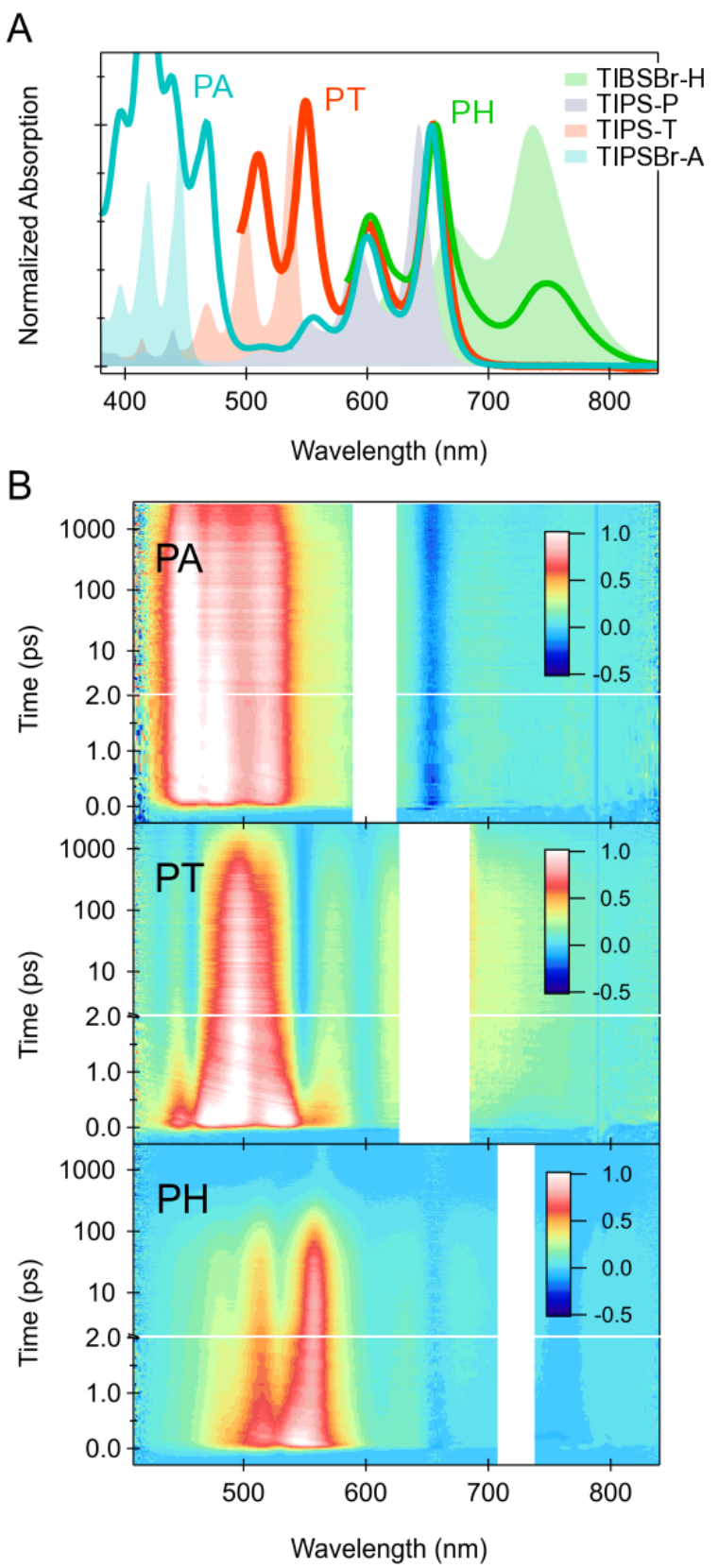

Figure 1. (A) Steady-state absorption spectra of PA, PT and PH, along with a TIPS-anthracene derivative, TIPS-tetracene, TIPS-pentacene and a TIBS-hexacene derivative. Absorption spectra are taken in chloroform and normalized at the pentacene absorption feature. (B) Transient absorption spectra of PA excited at $600 \mathrm{~nm}$, PT excited at $660 \mathrm{~nm}$ and PH excited at $730 \mathrm{~nm}$, at fluence of $25 \mu \mathrm{J} / \mathrm{cm}^{2}$ in chloroform. In each case, warmer colors represent increased absorption after excitation, and cooler colors represent decreased absorption.

The triplet pair features ground-state bleach (GSB) characteristics of both monomers in magnitudes corresponding to the relative absorption heights in the linear spectra, as expected for a triplet pair where both monomers are bleached.

The time constants for singlet fission $\left(\tau_{\mathrm{iSF}}\right)$ and triplet pair $\left(2 \mathrm{xT}_{1}\right)$ decay $\left(\tau_{2 \times \mathrm{T} 1}\right)$ are shown in Table 1 . Since there is no indication of a parasitic process that would compete with the singlet fission process, and the rates of SF are all orders of magnitude faster than fluorescence or internal conversion in PT and $\mathbf{P H}$, the data is consistent with a quantitative iSF process. In 
other words, the rates of singlet decay and triplet formation are directly correlated, and the yields are determined only by the kinetic competition between iSF and the intrinsic decay processes $(\sim 10 \mathrm{~ns}) .{ }^{[2 \mathrm{a}, 2 \mathrm{~d}, 8 \mathrm{a}, 8 \mathrm{c}]}$ This is in stark contrast to the dynamics observed in PA.

Table 1. Time constants for singlet fission $\left(\tau_{\text {iSF }}\right)$ and triplet pair recombination $\left(\tau_{2 \times \mathrm{TI}}\right)$ for the pentacene-tetracene $(\mathbf{P T})$ and pentacenehexacene (PH) heterodimers, compared to bipentacene (BP, homodimer).

\begin{tabular}{|c|c|c|}
\hline Compound $^{*}$ & $\boldsymbol{\tau}_{\text {ISC }}(\mathbf{p s})$ & $\boldsymbol{\tau}_{\mathbf{T} 1}(\mathbf{n s})$ \\
\hline PA & $1.0 \times 10^{5}$ & $1.74 \times 10^{4}$ \\
\hline iSF Compound & $\boldsymbol{\tau}_{\text {iSF }}(\mathbf{p s})$ & $\boldsymbol{\tau}_{\mathbf{2 \times T 1}}(\mathbf{n s})$ \\
\hline PT & 0.83 & 2.4 \\
\hline BP & 0.76 & 0.45 \\
\hline PH & 1.2 & 0.21 \\
\hline
\end{tabular}

${ }^{*}$ Compound PA: $\mathrm{S}_{1}$ lifetime $=11.5 \mathrm{~ns}, \sim 10 \%$ T yield.

Beyond the kinetics, the heterodimers enable us to probe the spatial dynamics of iSF since, due to asymmetry, the relative spectral weight of GSB in $\mathbf{P}$ and $\mathbf{T}$ will change when converting between different exciton states. Even though we qualitatively describe the absorption spectra of the heterodimers as combinations of the absorption features due to the individual monomers, when we pump the longer-wavelength absorption in any of the heterodimers both ground-state absorptions are bleached, although the longer-wavelength absorption is bleached more thoroughly than the shorter-wavelength absorption. This asymmetry in bleaching is in contrast to quantitative bleaching of both chromophores in bipentacenes, and it arises from the greater portion of the excited singlet wavefunction residing on the monomer unit that is associated with the lower-energy excited state. ${ }^{[2 \mathrm{~d}, 2 \mathrm{f}]}$ Averaging over vibrational and rotational degrees of freedom in the ensemble of molecules can thus lead to some partial bleaching (not quantitative, but non-zero) of the higher singlet-energy chromophore in the singlet.

In order to characterize the triplet pair, we compare singlet fission studies with sensitization experiments, in which the triplet states are populated in the heterodimers via collisional transfer from a triplet donor (anthracene) in excess concentration (Figure 2 ). In the case of the heterodimers, the anthracene can collide with and populate a triplet on either monomer. Interestingly, we do not observe any triplet transfer, despite the inequivalent triplet energies of the monomers. Presumably, the triplets cannot transfer in the heterodimers reported here because of the absence of the significant wavefunction overlap required for Dexter energy transfer, due to the highly-localized nature of acene triplets (further details in SI). ${ }^{[2 d, 12]}$ Given the pump energy employed in the sensitization experiments, individual molecules contain just one triplet exciton. Therefore, the spectra of individual triplets would appear significantly different from the triplet pair spectra produced by iSF. However, the ensemble contains a roughly even number of triplets on each monomer and can therefore be compared to iSF, which generates triplet pairs (further details in SI).

The photoinduced absorption (PIA) spectra of the $T_{1}$ resulting from sensitization and $2 \mathrm{xT}_{1}$ resulting from singlet fission are similar, but not identical. Modest spectral shifts of magnitude and/or wavelength of the PIA are found, consistent with reports of directly coupled pentacene dimers. ${ }^{[2 \mathrm{~d}-\mathrm{f}]}$ These shifts result from the strong correlation of the triplet pair when in close proximity, as demonstrated previously. ${ }^{[2 \mathrm{~d}]}$ While these spectra are similar, the dynamics are significantly different. In general, the triplet pairs produced from iSF tend to decay on much shorter timescales than individual triplets. In the case of $\mathbf{P T}$ and $\mathbf{P H}$, the lifetime of the $2 \mathrm{xT}_{1}$ is less than $3 \mathrm{~ns}$, as opposed to tens of microseconds for their individual $\mathrm{T}_{1} \cdot{ }^{[\mathrm{a}, 8 \mathrm{c}, 11]}$ The correlated triplet pair decay is apparent since both the pentacene and tetracene GSB signals decay at the same rate.
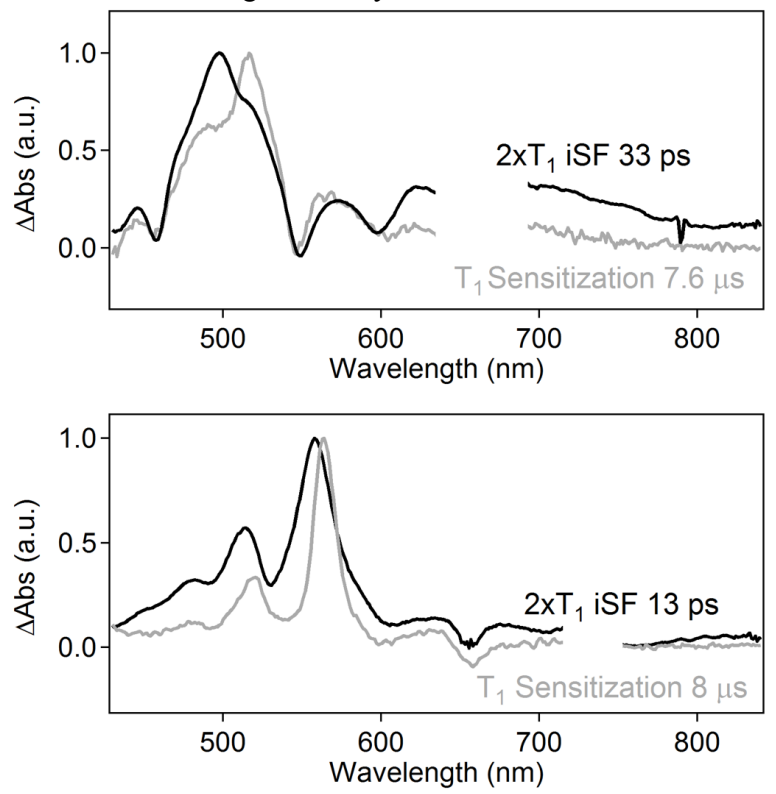

Figure 2. Comparison of triplet transient absorption spectra obtained by photosensitization (single $\left.\mathrm{T}_{1}\right)$ and singlet fission $\left(2 \mathrm{xT}_{1}\right)$ in $\mathbf{P T}$ and $\mathbf{P H}$.

While energetics have a dramatic impact on whether or not iSF will occur, the rates of iSF for PT, BP, and $\mathbf{P H}$ are surprisingly insensitive to the driving force, each being $\sim 1 p s$. In contrast, the recombination kinetics have a clear dependence on overall triplet pair energy. The lifetime of the triplet pair decreases following the trend, $\mathbf{P T}>\mathbf{B P}>\mathbf{P H}$, in agreement with the trend of decreasing energy of the triplet state (Figure 3 ). The triplet pair lifetime varies from $0.21 \mathrm{~ns}$ to $2.4 \mathrm{~ns}$ as the expected triplet pair energy decreases from $\sim 2.0 \mathrm{eV}$ to $\sim 1.3 \mathrm{eV}$. In all cases, the triplet pair is significantly less emissive than the singlet, and these lifetimes are much shorter than the radiative lifetime of the monomers. Therefore, the decay in the directly linked acene series is primarily non-radiative and can be explained by invoking a simple energy gap argument for non-radiative decay processes, where the rate of such a multiphonon process is inversely proportionate to the number of photons needed, i.e. the energy above the ground state. ${ }^{[9]}$

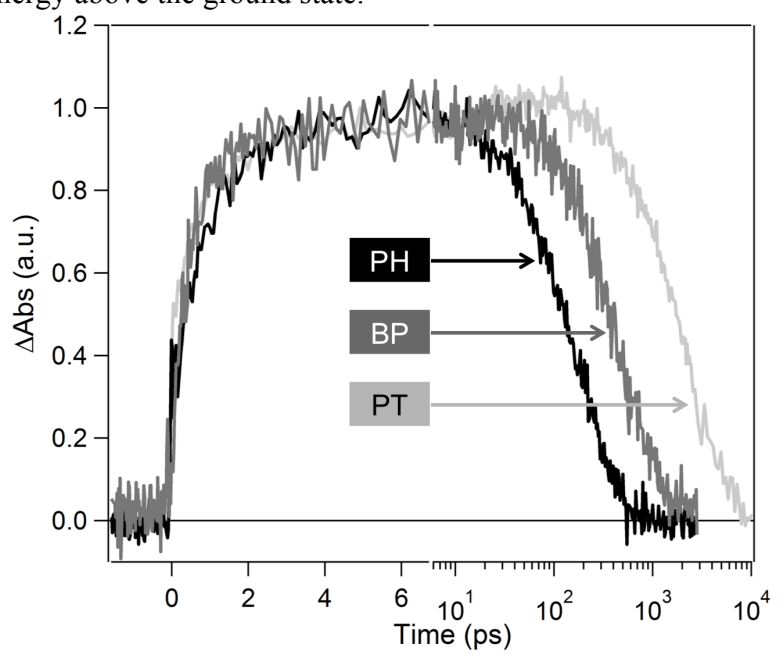

Figure 3. Rise and decay kinetic traces of the triplet pair in $\mathbf{P H}, \mathbf{B P}$, and PT, probed at the $\lambda_{\max }$ of the triplet excited state absorption spectra (683, $712,707 \mathrm{~nm}$ respectively). 
In summary, we have synthesized and characterized a series of pentacene-oligoacene heterodimers. In PA, iSF is significantly endothermic and does not occur. Instead, the compound undergoes the slow singlet state deactivation processes of internal conversion, fluorescence and a small amount of ISC. However, in PT and $\mathbf{P H}$, where iSF is energetically feasible, iSF occurs with $\sim 1$ ps time constant, as demonstrated by ultrafast transient absorption spectroscopy and triplet photosensitization experiments. Triplet pair recombination adheres to the energy gap law, but formation of the triplet pair appears to be insensitive to the driving force in iSF heterodimers. This study opens up a new possibility to tune the excited state dynamics of singlet fission in oligoacenes. Additionally, these heterodimers offer a broadly applicable strategy for creating iSF materials where energetics can be tuned and spectrally resolved monomer transitions can be exploited for detailed mechanistic studies of singlet fission.

Received: ((will be filled in by the editorial staff))

Published online on ((will be filled in by the editorial staff))

Keywords: singlet fission - oligoacene dimers · photophysics · conjugated materials $\cdot$ heterodimers

References:

[1] a) M. C. Hanna, A. J. Nozik, J. Appl. Phys. 2006, 100, 074510 ; b) M. B. Smith, J. Michl, Annu. Rev. Phys. Chem. 2013, 64, 361-386; c) M. B. Smith, J. Michl, Chem. Rev. 2010, 110, 6891-6936; d) I. Paci, J. C. Johnson, X. Chen, G. Rana, D. Popović, D. E. David, A. J. Nozik, M. A. Ratner, J. Michl, J. Am. Chem. Soc. 2006, 128, 16546-16553; e) D. N.

Congreve, J. Lee, N. J. Thompson, E. Hontz, S. R. Yost, P. D Reusswig, M. E. Bahlke, S. Reineke, T. Van Voorhis, M. A. Baldo, Science 2013, 340, 334-337; f) J. Lee, P. Jadhav, P. D. Reusswig, S. R. Yost, N. J. Thompson, D. N. Congreve, E. Hontz, T. Van Voorhis, M. A. Baldo, Acc. Chem. Res. 2013, 46, 1300-1311.

[2] a) E. Busby, J. Xia, Q. Wu, J. Z. Low, R. Rong, J. R. Miller, X.-Y. Zhu, L. M. Campos, M. Y. Sfeir, Nat. Mater. 2014, 14 , 426-433; b) E. Busby, J. Xia, J. Z. Low, Q. Wu, J. Hoy, L. M. Campos, M. Y. Sfeir, J. Phys. Chem. B 2015, 119, 7644-7650; c) J. Z. Low, S. N. Sanders, L. M. Campos, Chem. Mater. 2015, 27, 5453-5463; d) S. N. Sanders, E. Kumarasamy, A. B. Pun, M. T. Trinh, B. Choi, J. Xia, E. J. Taffet, J. Z. Low, J. R. Miller, X. Roy, X. Y. Zhu, M. L. Steigerwald, M. Y. Sfeir, L. M. Campos, J. Am. Chem. Soc. 2015, 137, 8965-8972; e) J. Zirzlmeier, D. Lehnherr, P. B. Coto, E. T. Chernick, R. Casillas, B. S. Basel, M. Thoss, R. R. Tykwinski, D. M. Guldi, Proc. Natl Acad. Sci. 2015, 112, 5325-5330; f) S. Lukman, A. J. Musser, K. Chen, S. Athanasopoulos, C. K. Yong, Z. Zeng, Q. Ye, C. Chi, J. M. Hodgkiss, J. Wu, R. H. Friend, N. C. Greenham, Adv. Funct. Mater. 2015, 25, 5452-5461; g) O. P. Varnavski, N. Abeyasinghe, J. Aragó, J. J. Serrano-Perez, E. Ortí, J. T. López Navarrete, K. Takimiya, J. Casado, T. G. Goodson III, J. Phys. Chem. Lett. 2015, 6, 1375-1384.

[3] a) N. Monahan, X.-Y. Zhu, Annu. Rev. Phys. Chem. 2015, 66, 601-618; b) T. Zeng, R. Hoffmann, N. Ananth, J. Am. Chem. Soc. 2014, 136, 5755-5764; c) K. Aryanpour, T. Dutta, U. N. V. Huynh, Z. V. Vardeny, S. Mazumdar, Phys. Rev. Lett. 2015, 115,267401

[4] a) J. N. Mastron, S. T. Roberts, R. E. McAnally, M. E. Thompson, S. E. Bradforth, J. Phys. Chem. B 2013, 117, 15519-15526; b) S. T. Roberts, R. E. McAnally, J. N. Mastron, D. H. Webber, M. T. Whited, R. L. Brutchey, M. E. Thompson, S. E. Bradforth, J. Am. Chem. Soc. 2012, 134, 6388-6400; c) R. D. Pensack, A. J. Tilley, S. R. Parkin, T. S. Lee, M. M. Payne, D. Gao, A. A. Jahnke, D. Oblinsky, P.-F. Li, J. E. Anthony, D. S. Seferos, G. D. Scholes, J. Am. Chem. Soc. 2015; d) S. R. Yost, J. Lee, M. W. B. Wilson, T. Wu, D. P. McMahon, R. R. Parkhurst, N. J. Thompson, D. N.
Congreve, A. Rao, K. Johnson, M. Y. Sfeir, M. G. Bawendi, T. M. Swager, R. H. Friend, M. A. Baldo, T. Van Voorhis, Nat. Chem. 2014, 6, 492-497; e) A. D. Chien, A. R. Molina, N. Abeyasinghe, O. P. Varnavski, T. Goodson, P. M. Zimmerman, J. Phys. Chem. C 2015, 119, 28258-28268; f) P. M. Zimmerman, F. Bell, D. Casanova, M. Head-Gordon, $J$. Am. Chem. Soc. 2011, 133, 19944-19952; g) S. Matsika, X Feng, A. V. Luzanov, A. I. Krylov, J. Phys. Chem. A 2014, 118, 11943-11955; h) X. Feng, A. V. Luzanov, A. I. Krylov, J. Phys. Chem. Lett. 2013, 4, 3845-3852.

[5] a) E. C. Alguire, J. E. Subotnik, N. H. Damrauer, J. Phys. Chem. A 2015, 119, 299-311; b) P. J. Vallett, J. L. Snyder, N. H. Damrauer, J. Phys. Chem. A. 2013, 117, 10824-10838.

[6] a) A. M. Müller, Y. S. Avlasevich, K. Müllen, C. J. Bardeen, Chem. Phys. Lett. 2006, 421, 518-522; b) A. M. Müller, Y. S. Avlasevich, W. W. Schoeller, K. Müllen, C. J. Bardeen, J. Am. Chem. Soc. 2007, 129, 14240-14250.

[7] a) M. Trlifaj, Czech J Phys 1977, 27, 190-199; b) M. Charbr, D. F. Williams, Chem. Phys. Lett. 1977, 49, 599-603; c) N. E. Geacintov, J. Burgos, M. Pope, C. Strom, Chem. Phys. Lett. 1971, 11, 504-508.

[8] a) H. L. Stern, A. J. Musser, S. Gelinas, P. Parkinson, L. M. Herz, M. J. Bruzek, J. Anthony, R. H. Friend, B. J. Walker, Proc. Natl Acad. Sci. 2015, 112, 7656-7661; b) K. N. Houk, P. S. Lee, M. Nendel, J. Org. Chem. 2001, 66, 5517-5521; c) B. J. Walker, A. J. Musser, D. Beljonne, R. H. Friend, Nat. Chem. 2013, 5, 1019-1024; d) L. Yang, M. Tabachnyk, S. L. Bayliss, M. L. Böhm, K. Broch, N. C. Greenham, R. H. Friend, B. Ehrler, Nano Lett. 2014, 15, 354-358.

[9] a) J. S. Wilson, N. Chawdhury, M. R. A. Al-Mandhary, M. Younus, M. S. Khan, P. R. Raithby, A. Köhler, R. H. Friend, $J$. Am. Chem. Soc. 2001, 123, 9412-9417; b) J. V. Caspar, T. J. Meyer, J. Phys. Chem. 1983, 87, 952-957; c) R. Englman, J. Jortner, Mol. Phys. 1970, 18, 145-164.

[10] a) W. Fudickar, T. Linker, J. Am. Chem. Soc. 2012, 134 , 15071-15082; b) J. E. Anthony, D. L. Eaton, S. R. Parkin, Org. Lett. 2002, 4, 15-18; c) J. E. Anthony, J. S. Brooks, D. L. Eaton, S. R. Parkin, J. Am. Chem. Soc. 2001, 123, 9482-9483; d) M. M. Payne, S. R. Parkin, J. E. Anthony, J. Am. Chem. Soc. 2005, 127, 8028-8029; e) B. Purushothaman, S. R. Parkin, J. E. Anthony, Org. Lett. 2010, 12, 2060-2063.

[11] H. Angliker, E. Rommel, J. Wirz, Chem. Phys. Lett. 1982, 87, 208-212.

[12] D. L. Dexter, J. Chem. Phys. 1953, 21, 836-850. 